\title{
Enzymverstärkung zündet nicht
}

\author{
Das Bemühen um eine Optimierung der spezifischen Immuntherapie \\ (SIT) durch immunmodulierende Adjuvanzien geht weiter: Britische \\ Forscher untersuchten jetzt den Nutzen einer Enzymverstärkung.
}

\begin{abstract}
$\ddot{A}$ rzte der Universität Southampton behandelten in einer plazebokontrollierten Doppelblindstudie $166 \mathrm{~Pa}$ tienten, die im Mittel seit 20 Jahren an Heuschnupfen litten und schlecht auf die Gabe von Antihistaminika oder intranasalen Glukokortikoiden ansprachen. Alle Probanden reagierten im Pricktest auf Graspollen positiv

Die SIT bestand aus zwei Injektionen im Abstand von 8 bis 11 Wochen, 2 bis 4 Monate vor der Pollensaison. Die Verumgruppe erhielt eine Mischung aus Extrakten (Baum-, Grasund Getreidepollen, Pilzsporen, Katzen- und Hundehaare, Hausstaubmilben), die mit dem Enzym $\beta$-Glukuronidase sowie mit Zyklohexandiol und Protaminsulfat versetzt war. Die Plazebogruppe erhielt Kochsalzlösung.
\end{abstract}

6 bis 8 Wochen nach der zweiten Injektion prüften die Forscher die Sensitivität auf Graspollen durch Pricktest. Während der 12-wöchigen Pollensaison protokollierten die Probanden ihre Symptome. Das Ergebnis war eindeutig: keinerlei Unterschiede zwischen Plazebo- und Verum-Gruppe.

Fazit: Die vorliegende Untersuchung konnte keine Wirkung der so genannten enzympotenzierten Hyposensibilisierung mit niedrig konzentrierten Allergenextrakten unter Zusatz von $\beta$ Glukoronidase zeigen.

Radcliffe MJ et al. Enzyme potentiated desensitisation in treatment of seasonal allergic rhinitis: double blind randomised controlled study. Br Med J 2003; 327: 251-4

\section{Heftige Diskussion}

Die nebenstehend referierte Studie von Radcliffe et al. hat eine Reihe kritischer und lesenswerter Kommentare provoziert, die auf der Homepage des British Medical Journal unter der Adresse http://bmj.com/cgi/eletters/

327/7409/251\#35311 nachzulesen sind.

Die Kritik entzündet sich

— an der Selektion von Patienten, die nicht mehr auf symptomatische Therapien ansprachen,

- an der Verwendung niedrig dosierter, komplexer Allergenmischungen,

- an fehlenden Konzentrationsangaben für die Allergenextrakte,

- an der unverstandenen Wirkungsweise der angeblich immunmodulierenden Adjuvansmischung

— sowie an Mängeln in der statistischen Auswertung.

Es bleibt noch anzumerken, dass von den sieben zitierten Referenzstudien, in denen für kleine Testgruppen signifikante Wirkungen der enzymverstärkten SIT nachgewiesen werden konnten, nur zwei in renommierten Journalen erschienen sind.

\section{Tabletten statt Tropfen}

\section{Die sublinguale Immuntherapie (SLIT) wird von vielen Patienten besser akzeptiert als die klassische subkutane Form. Compliance- Probleme können sich noch ergeben, weil die Tropfen 2 Minuten lang unter der Zunge gehalten werden müssen. Sind Lutsch- tabletten eine Alternative?}

$\mathrm{P}$ atienten zwischen 7 und 55 Jahren mit saisonaler allergischer Rhinitis gegen Ragweed-Pollen erhielten von März bis Oktober entweder eine SLIT $(\mathrm{n}=55)$ oder eine Plazebobehandlung $(\mathrm{n}=55)$. Dabei wurden in der 28-tägigen Auftitrationsphase zunächst Tropfen gegeben, in einer 30-tägigen Übergangsphase erfolgte die weitere Dosisauftitration mit Tabletten. In der 5,5 Monate dauernden Erhaltungsphase während der Pollensaison nahmen die Patienten dann je nach Verträglichkeit pro Woche ein bis drei Ragweed-Tabletten (100 IR/Tablette), die bis zur vollständigen Auflösung im Mund gehalten wurden.
Über die gesamte Pollensaison hinweg wurde die Verumtherapie in der Gesamtbeurteilung durch Patienten und Arzt signifikant besser beurteilt als die Plazebogabe. Patienten, die die Maximaldosis von drei Tabletten pro Woche einnahmen $(n=26)$, erzielten in zahlreichen Wirksamkeitsparametern signifikant bessere Symptomreduktionen als Patienten unter niedrigeren Dosierungen. Bei der Einnahme von symptomatisch wirksamen Medikamenten ergab sich ein Trend zur Dosisreduktion unter Verum.

Immunologisch zeigte sich unter der SLIT ein signifikanter Anstieg der
IgE-Spiegel im Vergleich zu Plazebo $(+51 \%$ vs. $+30 \%, \mathrm{p}=0,0005)$ sowie auch ein Anstieg der IgG-Spiegel $(+56 \%$ vs. $+7 \%, \mathrm{p}<0,0001)$. Bei den Hautpricktests reduzierte sich unter SLIT die Reaktivität signifikant ( $\mathrm{p}<$ 0,03) gegenüber Plazebo.

Schwere Nebenwirkungen traten nicht auf, leichtere Nebenwirkungen wie sublinguales Brennen wurden in der Verumgruppe häufiger registriert ( $\mathrm{p}<0,0001)$. Nach Dosisanpassung verschwanden diese wieder.

Fazit: Die Studie belegt Wirksamkeit und Sicherheit einer SLIT mit Ersatz der Tropfen durch eine Tablettenformulierung in. Der Effekt der SLIT ist dosisabhängig.

\section{$b k$}

André $\mathbf{C}$ et al. A double-blind placebocontrolled evaluation of sublingual immunotherapy with a standardized ragweed extract in patients with seasonal rhinitis. Int Arch Allergy Immunol 2003; 131: 111-8 